\title{
Vorticity and polarization in baryon rich matter
}

\section{Baznat}

Institute of Applied Physics, Academy of Sciences of Moldova, MD-2028 Kishinev, Moldova

E-mail: baznatetheor.jinr.ru

\section{K. Gudima}

Institute of Applied Physics, Academy of Sciences of Moldova, MD-2028 Kishinev, Moldova

E-mail: gudima@cc.acad.md

\section{A. Sorin*}

JINR, 141980, Dubna, Russia

E-mail: sorin@theor.jinr.ru

\section{O. Teryaev}

JINR, 141980, Dubna, Russia

E-mail: teryaev@theor.jinr.ru

The polarization of hyperons due to axial chiral vortical effect is discussed. The effect is proportional to chemical potential squared and is pronounced at lower energies in baryon-rich matter. The polarization of antihyperons has the same sign and larger magnitude. The emergence of vortical structures is observed in kinetic QGSM models. The hydrodynamical helicity separation receives the contribution of longitudinal velocity and vorticity implying the quadrupole structure of the latter. The transition from the quark vortical effects to baryons in confined phase may be achieved by exploring the axial charge.

Critical Point and Onset of Deconfinement - CPOD2017

7-11 August, 2017

The Wang Center, Stony Brook University, Stony Brook, NY

* Speaker. 


\section{Introduction}

The experimental evidences for polarization of hyperons in heavy-ion collisions found by STAR collaboration [1] attracted recently much attention $[2,3,4,5,6]$.

The studies of polarization are often performed [7] in the framework of approach exploring local equilibrium thermodynamics [8] and hydrodynamical calculations of vorticity $[9,10,11]$.

There is another approach to polarization first proposed in [12] and later in [13]. The so-called axial vortical effect (see e.g. [14] and references therein) being the macroscopic manifestation of axial anomaly [15] leads to induced axial current of strange quarks which may be converted to polarization of $\Lambda$-hyperons $[12,13]$.

The effect is proportional to vorticity and helicity of strongly interacting medium which was calculated [16] in the kinetic Quark-Gluon-String Model(QGSM)[17, 18, 19]. In this way, in fact, some particular case of fundamental relation between kinetic and hydrodynamic description was considered.

This calculation was later made more detailed [20], including the spatial and temporal dependence of strange chemical potential which is also the ingredient of anomalous approach to polarization.

\section{Helicity separation and quadrupole structure}

The effect of helicity separation was found in [16]. It was also found [16] that it receives contributions from both transverse and longitudinal velocity and vorticity. The latter, as the longitudinal velocity changes sign in each half-space, should lead to the quadrupole structure of longitudinal vorticity.

Indeed, the longitudinal vorticity averaged over $z$ in the symmetric cylinder $-z, z$ should change sign when $x \rightarrow-x$ in order to have non-zero helicity in the lower and upper half-planes. Also, it should change the sign when $y \rightarrow-y$ in order to guarantee that the signs of helicity in these half-planes are different.

We now supplement this conclusion following already from observations in [16] by explicit calculation (Fig.1).

\section{Anomalous mechanism of hyperon polarization}

Anomalous mechanism of polarization makes this effect qualitatively similar to the local violation [21] of discrete symmetries in strongly interacting QCD matter. The most well known here is the Chiral Magnetic Effect (CME) which uses the (C)P-violating (electro)magnetic field emerging in heavy ion collisions in order to probe the (C)P-odd effects in QCD matter.

The polarization, in turn, is similar to Chiral Vortical Effect (CVE)[22] due to coupling to P-odd medium vorticity leading to the induced electromagnetic and all other conserved-charge currents [12], in particular the baryonic one.

Recently the pioneering preliminary results on global polarization of $\Lambda$ and $\bar{\Lambda}$ hyperons in $A u-A u$ collisions in Beam Energy Scan at RHIC were released [1] and the qualitative tendency of polarization decrease with energy in agreement with the prediction [12] was revealed. The 


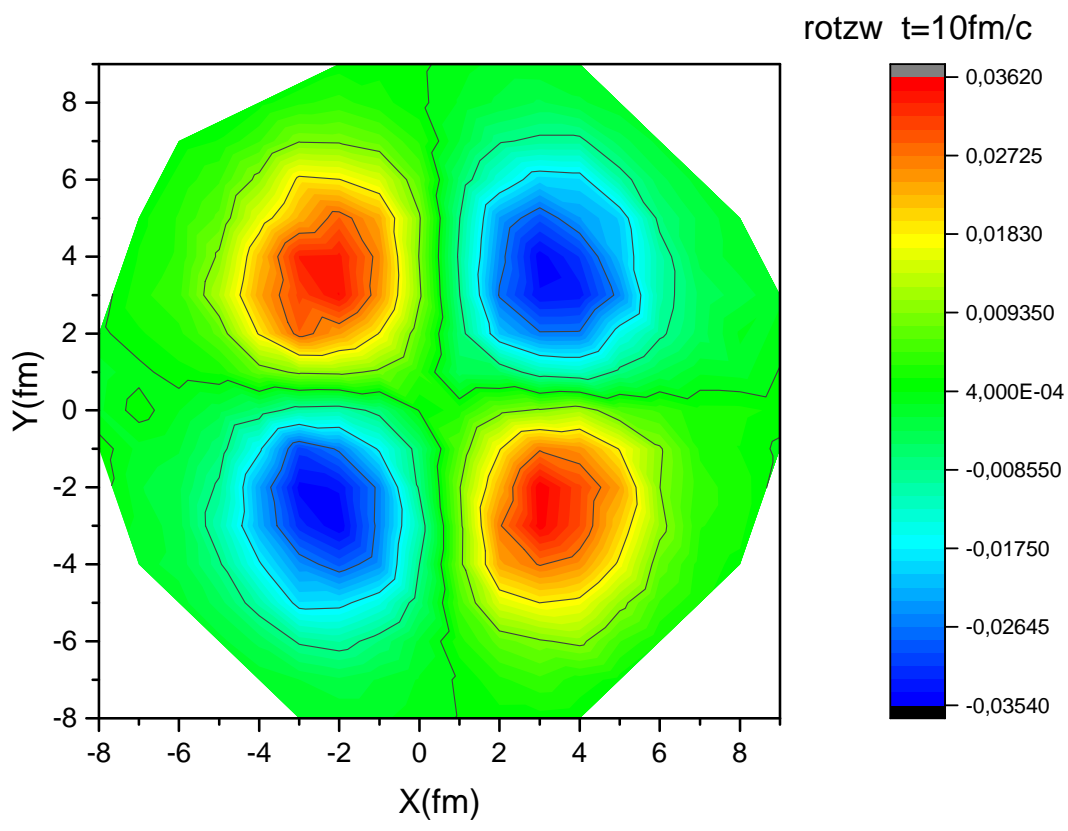

Figure 1: Quadrupole structure of longitudinal vorticity.

recent theoretical analysis [23] suggested that decrease of polarization with energy is related to the suppression of Axial Magnetic effect contribution in strongly correlated QCD matter found in lattice simulations.

Indeed, the chiral vorticity coefficient describing the axial vortical effect

$$
c_{V}=\frac{\mu_{s}^{2}+\mu_{A}^{2}}{2 \pi^{2}}+\frac{T^{2}}{6},
$$

contains temperature dependent term related to gravitational anomaly [24], and naively it can be quite substantial and increase with energy. However, lattice simulations [25] lead to the zero result in the confined phase and to the suppression by one order of magnitude at high temperatures. Neglecting axial chemical potential, the coefficient $c_{V}$ takes the form

$$
c_{V}=\frac{\mu_{s}^{2}}{2 \pi^{2}}+k \frac{T^{2}}{6},
$$

As soon as for free fermion gas the $T^{2} / 6$ term is recovered [26] for large lattice volume at fixed temperature, the above-mentioned suppression should be attributed to the correlation effects. It was suggested [23], that the accurate measurements of polarization energy dependence may serve a sensitive probe of strongly correlated QCD matter. Here we discuss the numerical simulations to implement this suggestion.

\section{Numerical simulations of axial anomaly contributions to (anti)hyperon polarization}

We performed the numerical simulations in QGSM model $[17,18,19]$. We decomposed the 
space-time to the cells, allowing to define velocity and vorticity in the kinetic model, as described in detail in [16]. To define the strange chemical potential (assuming that $\Lambda$ polarization is carried by strange quark) we used the matching procedure [20] of distribution functions to its (local) equilibrium values. We also determine in this way the values of temperature [27]. In general, let us stress that we realized in our particular case the relation between kinetics, hydrodynamics and thermodynamics.

We first neglect the gravitational anomaly contribution and start by considering the energy dependence of polarization (described in detail in [23]) for three values of impact parameter. The results are presented at Fig.2. The curves correspond to $b=8.0 \mathrm{fm}, 6.4 \mathrm{fm}, 4.8 \mathrm{fm}$.

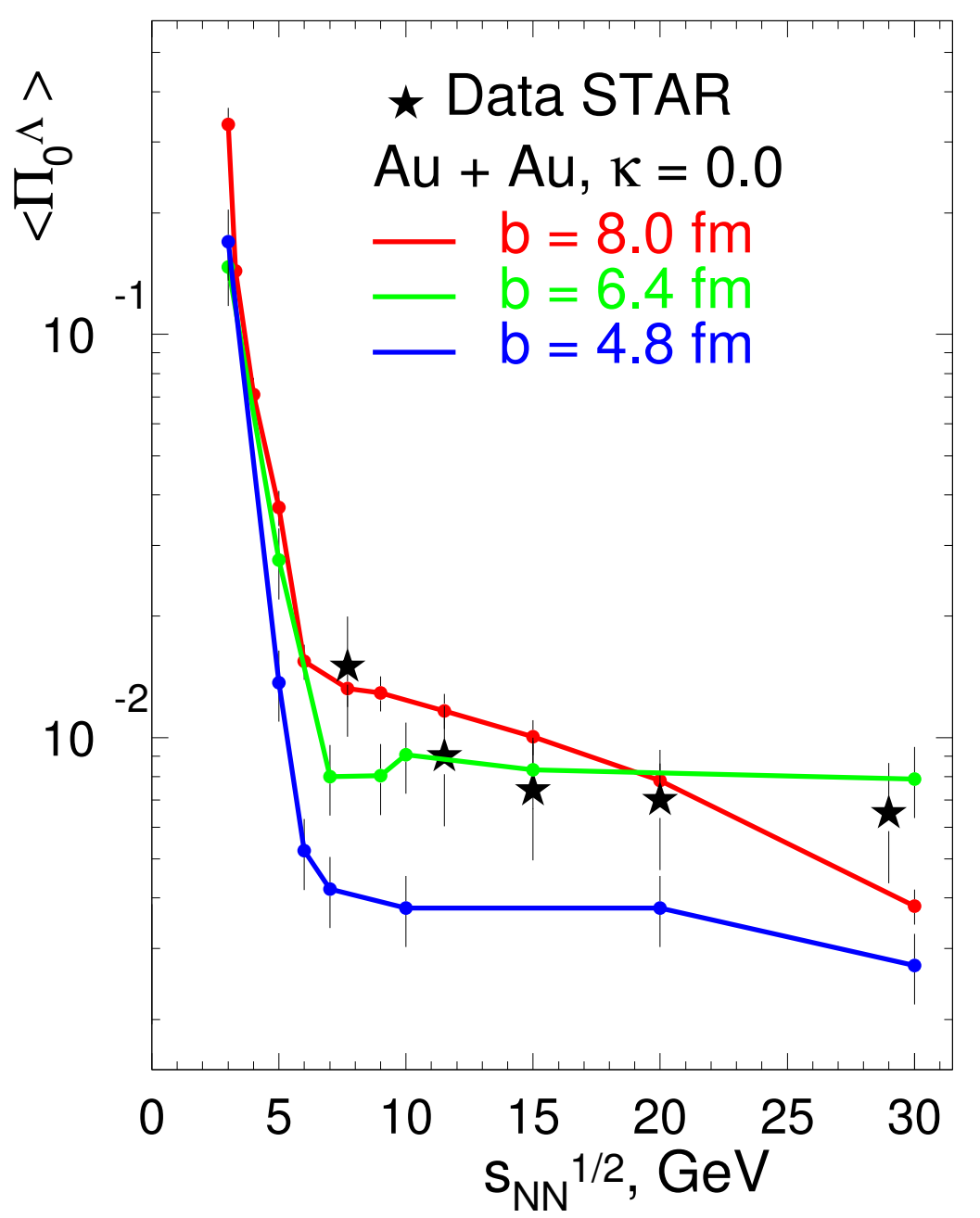

Figure 2: Energy dependence of polarization for different values of impact parameter.

We continue by inclusion of the contribution related to the gravitational anomaly [27]. The results are presented at Fig.3. We consider as a starting point the original value of anomaly coeffi- 
cient [24] $T^{2} / 6$ which is reproduced for large lattice volume at fixed temperature [26]. We present the curves following from the coefficients suppressed by factor $k$ (3.2) resulting from the lattice calculations [25]. We compare values of $k=1$ with $k=0,1 / 15,1 / 10$. As one can see, the latticesupported value $1 / 15$ is most close to the behavior of preliminary data which may be considered as a signal of strongly correlated matter formation. The closeness of $k=0$ curve to the experimental points may be related to the contribution of confinement phase, where lattice calculations [25] lead to zero temperature-dependent effect. At the same time, already $k=1 / 10$ leads to the curve growing with energy, while $k=1$ leads to extremely strong growth.

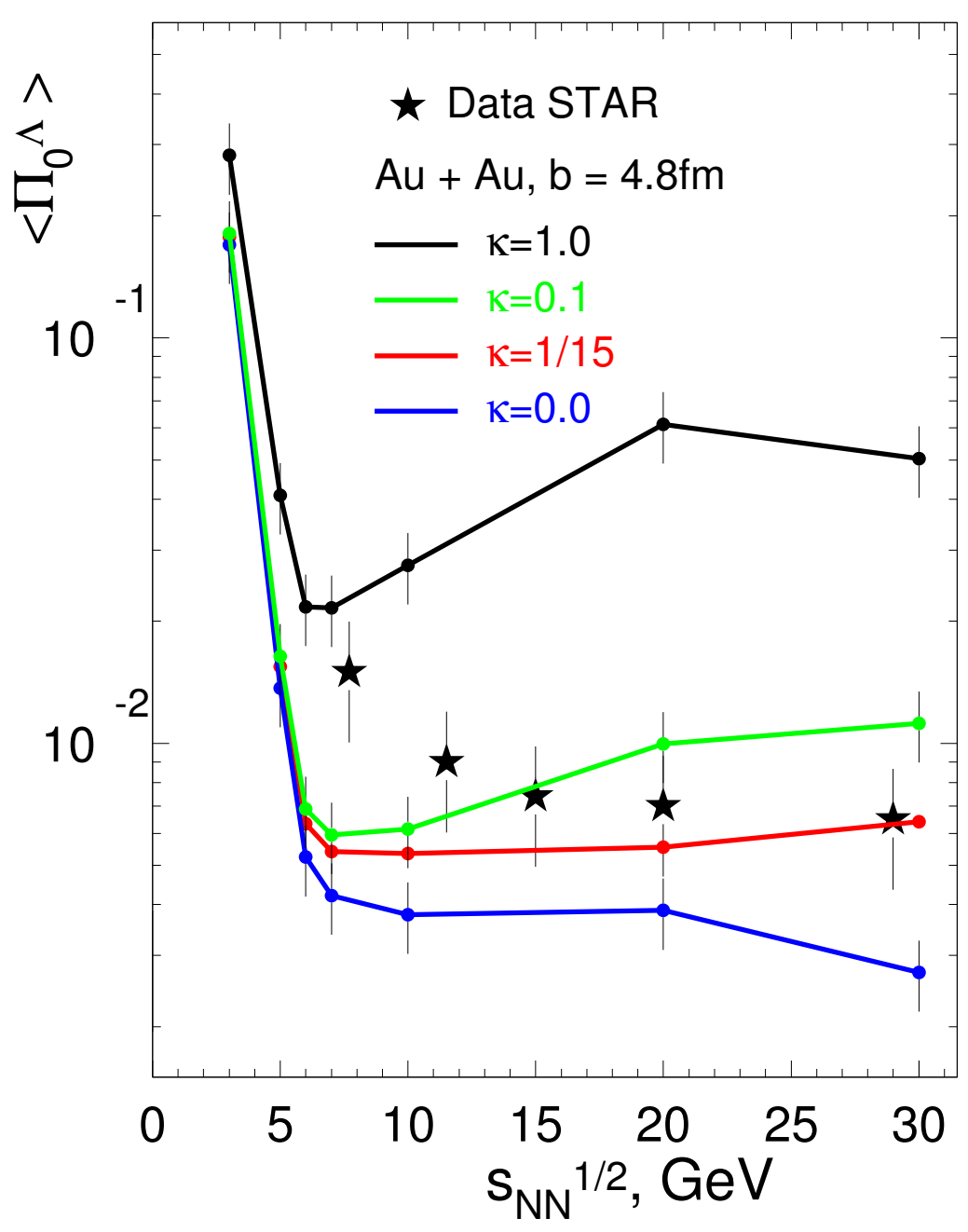

Figure 3: Energy dependence of the polarization for different values of the gravitational anomaly contribution.

The $\bar{\Lambda}$ polarization emerges due to the polarization of $\bar{s}$-quarks, which has the same sign, as the axial current and charge are C-even operators. The magnitude of the $\bar{\Lambda}$ polarization is larger 
as the same axial charge is distributed between the polarizations of the smaller number of particles [23]. It is mandatory to take into account the contribution of $K^{*}$ mesons. In the case of $\Lambda$ the $K^{*-}, \bar{K}^{0}$ mesons contain two sea(anti)quarks and does not change the polarization significantly. At the same time, for $\bar{\Lambda}$ the relevant $K^{*+}, K^{0}$ mesons are more numerous and make the excess of $\bar{\Lambda}$ polarization less pronounced.

Note that this excess is larger for smaller energies, where suppression of $\bar{\Lambda}$ is larger. This differs from the (C-odd) effect of magnetic field, which is increasing with energy, although more detailed studies taking into account the finite time of magnetic field existence are required.

The quantitative analysis of these effects, taking into account the gravitational anomaly contribution, is presented at Fig.4. The result is in reasonable agreement with STAR data, although

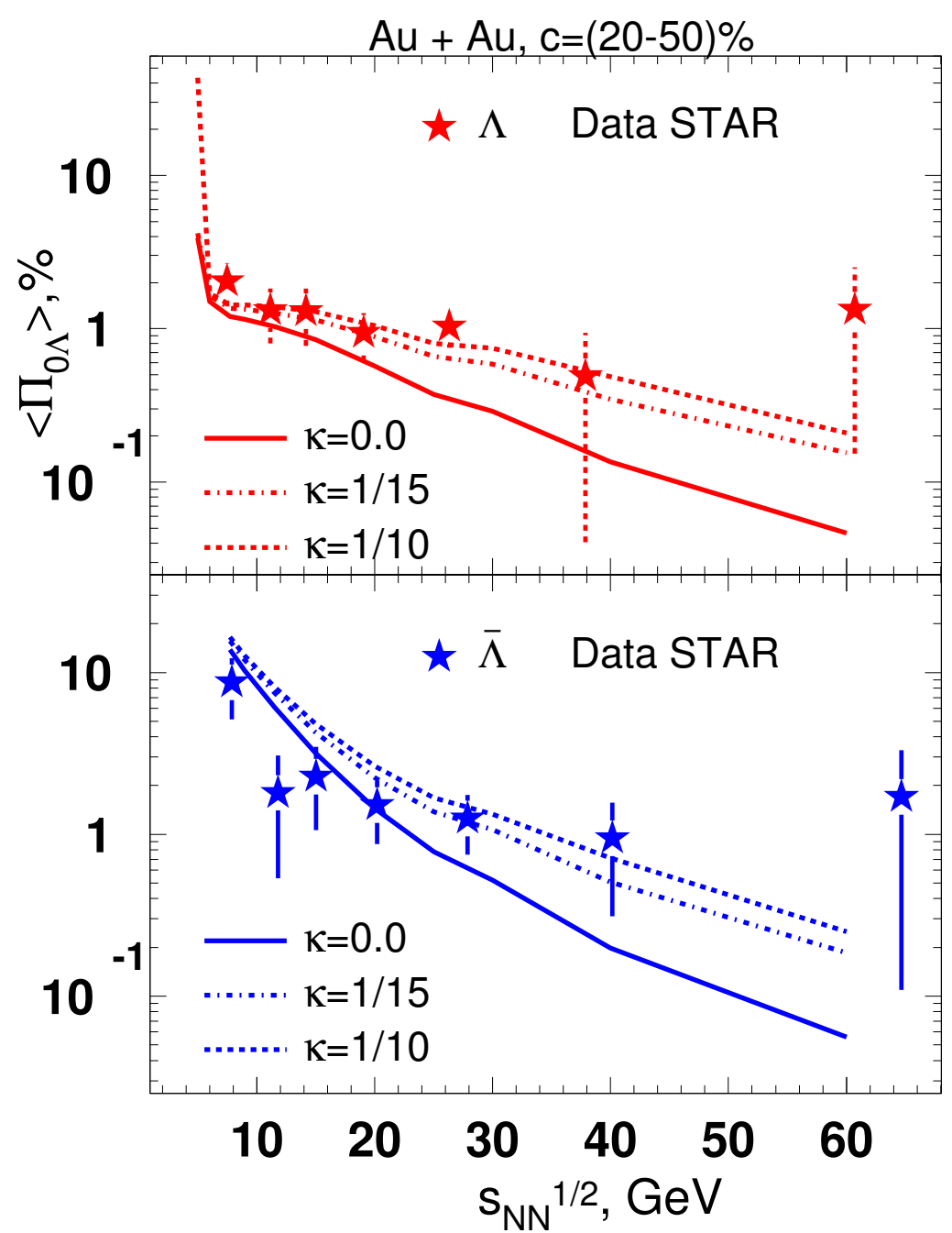

Figure 4: Comparison of $\Lambda$ and $\bar{\Lambda}$ polarizations for different values of gravitational anomaly contribution. further analysis is required. 


\section{Conclusions}

We found that the anomalous mechanism may naturally explain:

- Decrease of polarization with energy.

- The polarization of antihyperons of the same sign and larger magnitude as hyperons with the difference dereasing with energy.

Further theoretical and experimental studies should be performed. In particular, this is related to the polarization of other $(\Sigma, \Xi)$ hyperons, which should have the same (up to the mass rescaling) polarization in thermodynamical approach, but may differ significantly due to the different chemical potentials in the anomalous approach.

\subsection{Acknowledgments}

A.S. is indebted to the CPOD2017 Organizers for warm hospitality. The work was supported by RFBR grant 17-02-01108.

\section{References}

[1] L. Adamczyk et al. [STAR Collaboration], Nature 548, 62 (2017) doi:10.1038/nature23004 [arXiv:1701.06657 [nucl-ex]].

[2] F. Becattini, I. Karpenko, M. Lisa, I. Upsal and S. Voloshin, Phys. Rev. C 95, no. 5, 054902 (2017) doi:10.1103/PhysRevC.95.054902 [arXiv:1610.02506 [nucl-th]].

[3] I. Karpenko and F. Becattini, Eur. Phys. J. C 77, no. 4, 213 (2017) doi:10.1140/epjc/s10052-017-4765-1 [arXiv:1610.04717 [nucl-th]].

[4] I. Karpenko and F. Becattini, J. Phys. Conf. Ser. 779, no. 1, 012068 (2017) doi:10.1088/1742-6596/779/1/012068 [arXiv:1611.08136 [nucl-th]].

[5] Y. Xie, R. C. Glastad and L. P. Csernai, Phys. Rev. C 92 (2015) no.6, 064901 doi:10.1103/PhysRevC.92.064901 [arXiv:1505.07221 [nucl-th]].

[6] R. h. Fang, J. y. Pang, Q. Wang and X. n. Wang, arXiv:1611.04670 [nucl-th].

[7] F. Becattini, L. Csernai and D. J. Wang, " $\Lambda$ polarization in peripheral heavy ion collisions," Phys. Rev. C 88, no. 3, 034905 (2013) [arXiv:1304.4427 [nucl-th]].

[8] F. Becattini, L. Bucciantini, E. Grossi and L. Tinti, Eur. Phys. J. C 75, no. 5, 191 (2015) doi:10.1140/epjc/s10052-015-3384-y [arXiv:1403.6265 [hep-th]].

[9] B. Betz, M. Gyulassy and G. Torrieri, Phys. Rev. C 76 (2007) 044901 doi:10.1103/PhysRevC.76.044901 [arXiv:0708.0035 [nucl-th]].

[10] L. P. Csernai, V. K. Magas and D. J. Wang, Phys. Rev. C 87 (2013) no.3, 034906 doi:10.1103/PhysRevC.87.034906 [arXiv:1302.5310 [nucl-th]].

[11] L. P. Csernai, D. J. Wang and T. Csorgo, Phys. Rev. C 90 (2014) no.2, 024901 doi:10.1103/PhysRevC.90.024901 [arXiv:1406.1017 [hep-ph]].

[12] O. Rogachevsky, A. Sorin and O. Teryaev, "Chiral vortaic effect and neutron asymmetries in heavy-ion collisions," Phys. Rev. C 82, 054910 (2010) [arXiv:1006.1331 [hep-ph]]. 
[13] J. -H. Gao, Z. -T. Liang, S. Pu, Q. Wang and X. -N. Wang, "Chiral Anomaly and Local Polarization Effect from Quantum Kinetic Approach,” Phys. Rev. Lett. 109, 232301 (2012) [arXiv:1203.0725 [hep-ph]].

[14] T. Kalaydzhyan, "Temperature dependence of the chiral vortical effects," Phys. Rev. D 89, no. 10, 105012 (2014) [arXiv:1403.1256 [hep-th]].

[15] D.T. Son and P. Surowka, Phys. Rev. Lett. 103, 191601 (2009)

[16] M. Baznat, K. Gudima, A. Sorin and O. Teryaev, "Helicity separation in Heavy-Ion Collisions," Phys. Rev. C 88, 061901 (2013) [arXiv:1301.7003 [nucl-th]].

[17] V.D. Toneev, K.K. Gudima, Nucl. Phys. A 400, 173c (1983).

[18] V.D. Toneev, N.S. Amelin, K.K. Gudima, S.Yu. Sivoklokov, Nucl. Phys. A 519, 463c (1990).

[19] N.S. Amelin, E.F. Staubo, L.S. Csernai et al., Phys.Rev. C 44, 1541 (1991).

[20] M. I. Baznat, K. K. Gudima, A. S. Sorin and O. V. Teryaev, Phys. Rev. C 93, no. 3, 031902 (2016) doi:10.1103/PhysRevC.93.031902 [arXiv:1507.04652 [nucl-th]].

[21] K. Fukushima, D.E. Kharzeev and H.J. Warringa, “The Chiral Magnetic Effect,” Phys. Rev. D 78, 074033 (2008) [arXiv:0808.3382 [hep-ph]].

[22] D. Kharzeev and A. Zhitnitsky, “Charge separation induced by P-odd bubbles in QCD matter," Nucl. Phys. A 797, 67 (2007) [arXiv:0706.1026 [hep-ph]].

[23] A. Sorin and O. Teryaev, Phys. Rev. C 95, no. 1, 011902 (2017) doi:10.1103/PhysRevC.95.011902 [arXiv:1606.08398 [nucl-th]].

[24] K. Landsteiner, E. Megias, L. Melgar and F. Pena-Benitez, "Holographic Gravitational Anomaly and Chiral Vortical Effect," JHEP 1109 (2011) 121

[25] V. Braguta, M.N. Chernodub, K. Landsteiner, M.I. Polikarpov, M.V. Ulybyshev Phys.Rev. D88 (2013) 071501 DOI: 10.1103/PhysRevD.88.071501 e-Print: arXiv:1303.6266 [hep-lat]; V. Braguta, M.N. Chernodub, V.A. Goy, K. Landsteiner, A.V. Molochkov, M.I. Polikarpov, Phys.Rev. D89 (2014) no.7, 074510 DOI: 10.1103/PhysRevD.89.074510 e-Print: arXiv:1401.8095 [hep-lat]; V. Braguta, M. N. Chernodub, V. A. Goy, K. Landsteiner, A. V. Molochkov and M. Ulybyshev, "Study of axial magnetic effect," AIP Conf. Proc. 1701, 030002 (2016); doi:10.1063/1.4938608; V. Goy, "Investigation of SU(2) gluodynamics in the framework of the latice approach", PhD Thesis (in Russian), Vladivostok, 2015.

[26] P. V. Buividovich, "Axial Magnetic Effect and Chiral Vortical Effect with free lattice chiral fermions," J. Phys. Conf. Ser. 607, no. 1, 012018 (2015) doi:10.1088/1742-6596/607/1/012018 [arXiv:1309.4966 [hep-lat]].

[27] M. Baznat, K. Gudima, A. Sorin and O. Teryaev, arXiv:1701.00923 [nucl-th]. 